\title{
Recent development of scale marine aquaculture wastewater treatment
}

\author{
Panpan LIU ${ }^{1, a}$, Liping Qiu ${ }^{1, b^{*}}$, Shoubin Zhang ${ }^{1, c}$, Jiabin Wang ${ }^{1, d}$, Kang Xie ${ }^{1, e}$ \\ ${ }^{1}$ School of Civil Engineering and Architecture, University of Jinan, No.336. West Road of Nan \\ Xinzhuang. Jinan. 250022, PR China
}

a liupanpan_1012@163.com, blipingqiu@163.com, 'cea_zhangsb@ujn.edu.cn, 'cea_wangjb@ujn.edu.cn, ecea_xiek@ujn.edu.cn,

* Corresponding Author

Keywords: sea-water aquiculture; effluent treatment; marine environment

Abstract. With the rapid development of China's large-scale mariculture, the emissions of mariculture wastewater are increasing, which had already exceeded land-based sewage, and becoming a serious marine environmental problem, therefore, the treatment and control of large-scale mariculture wastewater have been more and more important. In this paper, recent development of large-scale mariculture in China was first introduced, and then, the characteristics of mariculture wastewater quality and quantity was analyzed as well as the mariculture wastewater treatment technology in the world was also reviewed. Finally, the development direction of China's future mariculture wastewater treatment technology and policy were predicted in order to provide references for the environmental protection and development of mariculture.

Seawater aquaculture has become an important source of protein in the world. With the increase of aquaculture scale and strength, the development of mariculture was increasing scale and industrialization, wastewater emissions increased rapidly and has a large amount of water, high strength and difficult to control, such as characteristics .A lot of mariculture wastewater into the sea water will lead to the seawater nutrient salt content increased rapidly, the abnormal reproduction of algae, the frequent occurrence of red tide and water in species diversity of damage, it has been bring serious pollution of the marine environment .

At present, many of our coastal aquaculture water were different degrees of deterioration of water quality, composed of large-scale mariculture because environmental problems gradually emerged. Therefore, to strengthen the protection of the marine environment, the systematic study of scale aquaculture wastewater treatment technology and countermeasures has become a top priority. This paper summarizes the development status of the marine aquaculture industry in our country. Characteristics of seawater aquaculture wastewater and the research status of domestic and overseas wastewater treatment, On the basis of this, the technology development strategy of marine aquaculture wastewater treatment was prospected in order to provide reference for the healthy and stable development of China's marine aquaculture industry.

\section{Development status of China's marine aquaculture industry}

As one of the most important industry in China, mariculture which is the substitution of traditional fishery resource has been greatly developed towards the direction of scale and industrialization since eighties of the $20^{\text {th }}$ century. In 2012, the total output value of mariculture is 17321.88 billion, with increase of 7915 millions compares to precious year. In recent years, the proportion of China total output of aquatic products in the world is about 30\% ranks first, China has become the largest production base and exporter in aquaculture production [1]. Mariculture plays an important role in our daily life, it can ensure food security of our food and greatly meets people's nutritional needs, improves people's diet structure and living standards, what is more the development and utilization of marine fishery resources has became one of national strategies.

However, large-scale aquaculture development and industrialization will inevitably bring a lot of wastewater and treatment, adding to the pollution load of aquiculture water and adjacent waters, 
threatening the sustainable development of marine environmental quality and marine fishery resources development industry. Due to "historical debts" and the rapid development aquaculture in recent years, China's marine aquaculture wastewater treatment is almost empty, related technology research lag, lack of management measures, resulting in the deterioration of the whole sea mariculture water quality of coastal waters, the red tide, diseases and other pollution incidents occur frequently, aquaculture resources shortage, fisheries production and quality decline, causing enormous economic losses. According to the "2010 China Fishery Statistics Yearbook" statistics, the total loss of aquatic products in China in 2009 due to marine pollution caused by the fishery disaster is 1.161 million tons and economic losses 15.227 billion Yuan.

Therefore, with the study mariculture wastewater treatment and emission control technologies, develop a new farming techniques and model that is friendly to environment has become a key problem that needs to be solved urgently in the scale of aquaculture.

\section{Water quality and quantity characteristics of seawater aquaculture wastewater}

The main pollutants in aquaculture wastewater are surplus food and biological metabolites, chemical drugs and therapeutic agents. One of the most important part is the breeding process in addition to excessive feed, when the food cannot be water aquaculture organisms in the full use of, the remaining part of the dissolved in water or to form sediment sink to the bottom of the pool, through continuous accumulation eventually lead to ocean, the water is polluted.

In addition, mariculture wastewater has the following characteristics : ( 1 )Mariculture wastewater with more emissions and less pollutant. ( 2 ) Compared with the industrial wastewater and domestic sewage, mariculture wastewater pollutants concentration is low, but the dissolved oxygen content is high. Its mass concentration is in commonly more than $5.0 \mathrm{mg} / \mathrm{L}$. Mariculture wastewater with low $\mathrm{C} / \mathrm{N}$, general for 3-10, far below the microbial optimal $\mathrm{C} / \mathrm{N}$ ratio. Because of the aquaculture wastewater has low $\mathrm{C} / \mathrm{N}$ and high dissolved oxygen content, Li Xiuchen et al [2] used the aquaculture solid waste as the carbon source in the seawater circulation farming system. Wastewater by hydrolysis and denitrification unit, effectively realized the deoxidization and organic carbon source added that makes the waste water purification effect is more significant. ( 3 ) Compared with common terrigenous sewage, mariculture wastewater pollutants will increase the difficulty of the processing technology and complexity in composition, salinity effect and ionic strength effect . ( 4 ) Mariculture wastewater needs strict processing. We should strictly control the relevant pollutant indexes and dissolved oxygen value, etc., because part of the wastewater could be used in aquaculture recycling 《Aquaculture industry standard of the People's Republic of China. Mariculture water emissions requirements (SC/T9103-2007) 》.

\section{Research status at home and abroad}

Current research situation in foreign countries. In recent years, some foreign scholars have recognized the necessity of mariculture wastewater treatment, the first began the study of mariculture wastewater treatment technology [3]. Currently, the main use of Recirculating Aquaculture Systems for mariculture wastewater in the international. RAS integrated use of sedimentation, filtration, biological wastewater treatment technology, sterilization and aeration temperature and a series of means, it will produce the breeding process of efficient treatment to remove pollutants in wastewater, so that the treated water can reach the level of recycling. Currently, the main use of Recirculating Aquaculture Systems for mariculture wastewater in the international. RAS integrated use of sedimentation, filtration, biological wastewater treatment technology, sterilization and aeration temperature and a series of means, it will produce the breeding process of efficient treatment to remove pollutants in wastewater, so that the treated water can reach the level of recycling. 
In addition to the RAS system, P. Babatsouli et al [4] study of single-stage treatment of saline wastewater developed a novel immobilized packed bed bioreactor, such light reactor can efficiently handle marine aquaculture wastewater. The results of the study showed that aquaculture wastewater treatment by 4 5h reactor, the removal of organic carbon, Nitrogen and Phosphorus were as high as 95\%. Merino et al [5] research aquaculture wastewater in the theoretical study on the settlement of particles, to effectively remove the suspended particulate matter in the aquaculture water provides the reliable theory basis. Comparison of Liu Changfa [6] studied the two settlement way of removal efficiency, found natural sedimentation sedimentation tank of nurseries in the wastewater of particulate matter removal rate was $69 \%$, and mechanical cyclone precipitation can remove the mariculture wastewater in more than $87 \%$ of the suspended solid particles matter.

In addition, the use of large algae and planktonic algae purification of aquaculture wastewater research and application is also more. Besides, in large algae and cultivation object of water, by controlling the algae of biomass can reduce the concentration of nutrients. University of Hawaii Wang design a composed of diatom (Chaetoceros) 、 shrimp and oysters of recirculating aquaculture system, diatoms can not only absorption in shrimp breeding generation of pollutants, also can be used as a bait of oysters. Because of the marine diatom has unique antibacterial ability, the method can also effectively prevent the occurrence of bacterial diseases in aquaculture object, also helps to reduce viral diseases [7]. El-Kassas [8] studies show that spirulina can not only provide food for shellfish, shrimp, can also significantly reduce the content of a farming system in ammonia and nitrate nitrogen in the system.

Domestic research status. At present, the domestic research on aquaculture wastewater treatment is still in the initial stage, some application research is carried out mainly around some specific management projects. The treatment difficulty of aquaculture wastewater was increased due to the effect of sea water salinity. The proprietary technology for the treatment of marine aquaculture wastewater is very rare. At present, we mainly use the conventional physical, chemical and biochemical process to treat aquaculture wastewater, the purpose is to reduce the chemical oxygen demand (COD), suspended matter (SS) and ammonia nitrogen $\left(\mathrm{NH}^{+}{ }_{4}-\mathrm{N}\right)$ concentration in aquaculture wastewater. Then part can be recycled.

$\mathrm{Xu}$ Yang et al [9] used biological filter reactor with filler of bamboo ring to process reticulating aquaculture wastewater from seawater fish factory achieved good treatment effect, specifically, the ammonia nitrogen removal efficiency of grouper fish biofilter was $27 \%-39 \%$ and the ammonia nitrogen removal efficiency of Kyong losses semilaevis biofilter was $20 \%-30 \%$, and nitrite nitrogen removal also had some effect.

Artificial Wetland can effectively remove suspended solids, nitrogen, phosphate, and other trace elements of wastewater, which have remarkable environmental benefits, ecological benefits and economic benefits, furthermore, the serviceability is broad[10].To achieve the purpose of contaminants removal, the interception and absorption of plant root system and the microbial metabolism in the system of artificial wetlands can be working. [11]. Li Huaizheng et al [12] preliminary resolve the pollution problem of water drainage in the park use through artificial slope Wetland / aquatic plant pond integrated technology to deal with saliferous wastewater, furthermore ,TN and TP of the strength reduction respectively reached $896 \mathrm{~kg} / \mathrm{hm}^{2}, 35.2 \mathrm{~kg} / \mathrm{hm}^{2}$ and $10.9 \mathrm{~kg} / \mathrm{hm}^{2}$ per year. GaoFeng et al [13] obtain better effect, in Kandelia candel artificial wetland purifying and circulating aquaculture wastewater, and the removal rate of $\mathrm{COD}$ and $\mathrm{NH}_{4}{ }^{+}-\mathrm{N}$ were $66.4 \%-73.8 \%$ and $64.3 \%-72.4 \%$, and which was significantly better than that with no plants.

\section{Conclusions and recommendations}

With population increasing and people's living standards continue to improve, the state attaches great importance to food safety, national health and ecological civilization construction, are increasingly high requirements on the development of aquaculture, mariculture development will by the size of the single type of to the environmental friendly and sustainable integrated ecological transition. However, at 
present our country is facing the seawater aquaculture wastewater treatment and emission control status is very serious, especially the serious lag of processing technology, has become a bottleneck restricting the development of marine resources.

First, put the marine aquaculture insurance into the policy of insurance. To promoting the policy agricultural insurance should also be some insurance premium subsidies given to the marine aquaculture insurance, enhancing the anti-risk ability of farmers. Secondly, in the future, we should learn from foreign experience, combined with the actual situation of the domestic aquaculture wastewater treatment, integrated metabolic engineering, fermentation engineering, biological technology and biological engineering technology, combination optimization process, research and development of highly purified, which suitable for China's national conditions of aquaculture wastewater treatment technology and process system, promote aquaculture wastewater recycling utilization, establish efficient circulation type high density aquaculture system, to reduce or avoid aquaculture wastewater discharge, the protection of the marine environment, reduce production costs and promote the seawater aquaculture industry sustainable development.

\section{Acknowledgements}

This study was partly supported by National Natural Science Foundation of China (51278225), Shandong Provincial Natural Science Foundation, China (ZR2013EEQ007, ZR2015EM021).

\section{References}

[1] Yusheng Chen, RuiJing Fang, Juan Qiao: Issues in Agricultural Economy, Vol. 6 (2012), p.72. In Chinese.

[2] Xiucheng Li, Lili Li: Transactions of the CSAE, Vol .26(2010). In Chinese.

[3] M.Shpigel, D. Ben-Ezra, et al: Aquaculture ,Vol . (2013), p.52.

[4] P. Babatsouli, S. Fodelianakis, N. Paranychianakis, et al: Journal of Hazardous Materials, Vol . 292(2015), p.155.

[5] G E. Merino, R H. Piedrahita, D E. Conklin: Aquacultural. Engineering ,Vol. 37(2007), p.79.

[6] Changfa Liu, Yan Xu,Zaisheng Yan, et al: Biochemical Engineering Journal, Vol. (2009) , p.1. In Chinese.

[7]J.K.Wang: Aquaculture engineering, Vol.28(2003), p.37.

[8] H Y. El-Kassas , A M M . Heneash , N R . Hussein : Journal of Genetic Engineering \& Biotechnology, Vol.(2015), p331.

[9] Yang Xu , Xi yang Gao, Yanqing Zhang, ZhiPei Liu, Ying Liu : Marine Science, Vol.34(2010), p26. In Chinese.

[10] X. Chen, A . C.Luo,K. Sato ,et al: Water and Environment Journal, Vol.23(2009), p255.

[11] A. Yalcuk , A . Ugurlu. Sato : Bioresource Technology, Vol.100(2009), p2521.

[12] Huaizheng Li,Yixing Zhang,Weibing Chen,Jianfeng Ye : ChinaWater \& Wastewater, Vol.27(2011), p56. In Chinese.

[13] Feng Gao , Chaohui Yang ,Chen Li, et al: Transactions of the Chinese Society of Agricultural Engineering, Vol.28(2012), p192. In Chinese. 